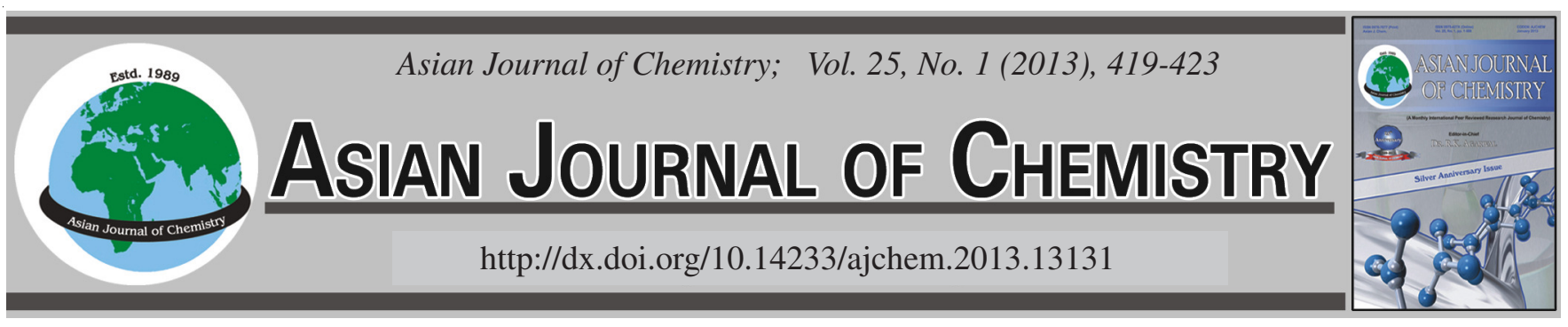

\title{
Diaryl Pyrazole-4-carbaldehyde Benzoylhydrazones Metal Complexes: Synthesis and Their Antibacterial and Antioxidant Screening
}

\author{
Muhammad Nasrullah ${ }^{1}$, Misbahul Ain Khan ${ }^{1}$, Muhammad Naeem Khan ${ }^{2, *}$, Mark G. Humphrey ${ }^{3}$, \\ Faizul Hassan Nasim ${ }^{1}$, Faryal Chaudhry ${ }^{1}$, Moeena Ghazal Abidi ${ }^{1}$, Umar Farooq $^{4}$ and Munawar Ali Munawar ${ }^{4}$
}

\begin{abstract}
${ }^{1}$ Department of Chemistry, The Islamia University of Bahawalpur, Bahawalpur, Pakistan ${ }^{2}$ Applied Chemistry Research Centre, PCSIR Laboratories Complex, Lahore, Pakistan ${ }^{3}$ Research School of Chemistry, Australian National University, Canberra, Australia ${ }^{4}$ Institute of Chemistry, University of the Punjab, Quaid-i-Azam Campus, Lahore, Pakistan
\end{abstract}

*Corresponding author: E-mail: changwani_1@yahoo.com compounds were characterized through elemental analysis and spectroscopic techniques (FTIR, ${ }^{1} \mathrm{H}$ NMR and Mass).

Key Words: Aryl pyrazoles, Vilsmeier-Haack reaction, Transition metal complexes, Antioxidant and antibacterial studies.

\section{INTRODUCTION}

Derivatives of pyrazole are widely used as ligands in coordination chemistry and are well known bioactive molecules. The presence of metal in their complexes enhances the bioactivity of these molecules. These metal based complexes have attracted considerable interest in chemistry and biology owing to their potentially beneficial biological activities which are attributed to a chelation phenomenon with trace of metal ions ${ }^{1-5}$. Hydrazones occupy unique position due to their diverse biological activity which has been recently reviewed ${ }^{6}$.

A number of hydrazones and their metal complexes have been synthesized and reported to exhibit biological activity ${ }^{7,8}$. In continuation of our previous studies of the metal complexes related to diphenylpyrazole compounds ${ }^{9-11}$, we report the synthesis of some novel metal complexes of diverse 1,3diarylpyrazole-4-carbaldehydes benzoylhydrazones.

\section{EXPERIMENTAL}

All the chemicals used in this study were of AR grade and were purchased from Merck and Aldrich and used without further purification. The ${ }^{1} \mathrm{H}$ NMR spectra were recorded on Varian MR Instrument at $300 \mathrm{MHz}$ in $\mathrm{CDCl}_{3}$ using tetramethylsilane (TMS) as internal standard. Electronspray ionization mass spectra (ESI MS) were recorded on VG AutoSpec M series (EBE) MS spectrometer. FTIR spectra were recorded on Bruker Tensor 27. Elemental analyses were performed using Carlo Erba 1106 automatic analyzer. Melting points were taken on a GallenKemp melting point apparatus and were uncorrected.

\section{Synthesis of ligands}

1,3-Diarylpyrazole-4-carbaldehydes (1-4): A mixture of an acetophenone $(12 \mathrm{~g}, 0.1 \mathrm{~mol})$ and phenylhydrazine $(10.8$ $\mathrm{g}, 0.1 \mathrm{~mol}$ ) in equimolar quantities along with a few drops of acetic acid in ethanol $(25 \mathrm{~mL})$ was heated for a few minutes on a hot plate to yield the corresponding acetophenone phenylhydrazone (Scheme-I). These hydrazones, without further purification, were treated with Vilsmeier-Haack reagent $\left(\mathrm{POCl}_{3}, \mathrm{DMF}\right)$. The temperature of the mixture was kept below $10{ }^{\circ} \mathrm{C}$ during addition, followed by heating on a water bath for $1 \mathrm{~h}$, poured into ice-cold water to give the precipitates of the corresponding carbaldehyde. The precipitates were washed with water and recrystallized from ethanol. The following 1,3-diarylpyrazole-4-carbaldehydes were prepared in this manner;

1,3-Diphenylpyrazole-4-carbaldehyde (1): m.p. 141-143 ${ }^{\circ} \mathrm{C}\left(\mathrm{Lit}^{12} .138{ }^{\circ} \mathrm{C}\right)$, yield; $7.4 \mathrm{~g} ; 90 \%$.

3-(4'-Chlorophenyl)-1-phenylpyrazole-4-carbaldehyde (2): m.p. $109-111^{\circ} \mathrm{C}\left(\mathrm{Lit}^{12} .110^{\circ} \mathrm{C}\right)$, yield; $7.5 \mathrm{~g} ; 88 \%$.

3-(4'-Nitrophenyl)-1-phenylpyrazole-4-carbaldehyde (3): m.p. $120-122{ }^{\circ} \mathrm{C}\left(\mathrm{Lit}^{12} .120{ }^{\circ} \mathrm{C}\right)$, yield; $6.9 \mathrm{~g} ; 81 \%$.

3-(4'-Methylphenyl)-1-phenylpyrazole-4-carbaldehyde (4): m.p. $160-162{ }^{\circ} \mathrm{C}\left(\mathrm{Lit}^{12} .165^{\circ} \mathrm{C}\right)$, yield; $6.9 \mathrm{~g} ; 85 \%$. 


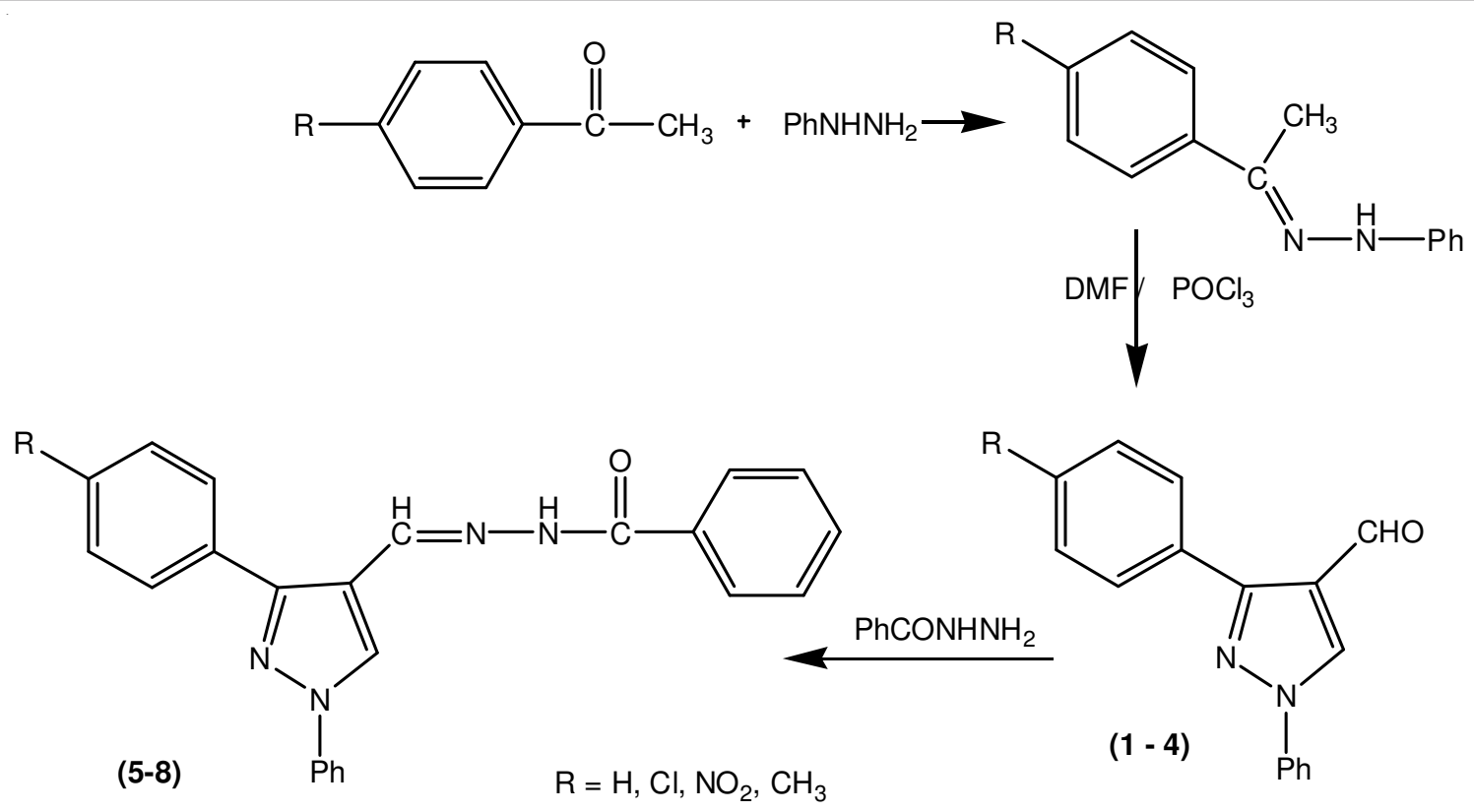

Scheme-I

1,3-Diarylpyrazole-4-carbaldehyde benzoylhydrazones (5-8): General method: A mixture of 1,3-diarylpyrazole-4carbaldehyde ( $5 \mathrm{mmol})$, benzhydrazide $(5 \mathrm{mmol})$ and 3-4 drops of phosphoric acid in ethanol $(30 \mathrm{~mL})$, was refluxed for $0.5 \mathrm{~h}$. After cooling, the mixture was poured into distilled water (150 $\mathrm{mL}$ ), off white precipitates were filtered off and recrystallized from ethanol.

The following hydrazones were prepared in this manner:

1,3-Diphenylpyrazole-4-carbaldehyde benzoylhydrazone (5): Off white crystals, m.p. $202{ }^{\circ} \mathrm{C}$, yield; $1.4 \mathrm{~g} ; 77 \%$.

3-(4'-Chlorophenyl)-1-phenylpyrazole-4-carbaldehyde benzoylhydrazone (6): Off-white crystals, m.p. $208^{\circ} \mathrm{C}$, yield; $1.7 \mathrm{~g} ; 85 \%$.

3-(4'-Nitrophenyl)-1-phenylpyrazole-4-carbaldehyde benzoylhydrazone (7): Yellow crystals, m.p. $204{ }^{\circ} \mathrm{C}$, yield; 1.7 g; $82 \%$.

3-(4'-Methylphenyl)-1-phenylpyrazole-4-carbaldehyde benzoylhydrazone $(\mathbf{8})$ : Off-white crystals, m.p. $201^{\circ} \mathrm{C}$. yield; $1.6 \mathrm{~g} ; 82 \%$.

Synthesis of metal complexes (9-32): General method: Metal(II) chlorides $(0.9 \mathrm{mmol})$ were dissolved in $(10 \mathrm{~mL})$ ethanol and the solution was mixed with a warm ethanolic solution $(20 \mathrm{~mL})$ containing equimolar quantity $(0.9 \mathrm{mmol})$ of ligands (5-8). The mixture was refluxed for $4 \mathrm{~h}$, cooled to room temperature and poured into distilled water $(150 \mathrm{~mL})$ to give crystalline precipitates. These were washed successively with water and ethanol, dried by vacuum suction and stored in a desiccator containing anhydrous calcium chloride. The physical and spectral data of complexes is tabulated in Tables 1 and 2.

\section{RESULTS AND DISCUSSION}

In continuation of our previous work on metal complexes of 1,3-diphenylpyrazole-4-carbonyl hydrazones of aryl aldehydes, we now report complexation studies of aroyl hydrazones of 1,3-diphenylpyrazole-4-carbaldehyde. To our best of knowledge, only one article has appeared describing the nickel complex of 1,3-diphenyl-4-carbaldehyde thiosemicarbazone ${ }^{13}$. The present work differs slightly from our previous ones with respect to the aldehydic and hydrazide functionalities which have been interchanged between the aryl and diphenylpyrazole moieties. The results are as follows.

Synthesis: Scheme-I gives the general method for the synthesis of the hydrazone ligands and their metal complexes with various bivalent metal ions. During the synthesis of aldehydes (1-4), it was found that some acetophenone phenylhydrazones intermediates were very unstable and decomposed on storage. Therefore, these were subjected to VilsmeierHaack reaction without further purification. The ligands (5-8) were obtained in good yields (75-80 \%) by the condensation of warm ethanolic solutions of corresponding aldehydes and benzhydrazide. Their formation was confirmed from their elemental analysis as well as mass and other spectra (Tables 1 and 2).

Various metal complexes (9-32) prepared during this work were also characterized through their elemental analysis and spectroscopic data. The metal contents of these complexes were determined with the help of AAS which showed that the complexes had a metal to ligand ratio of 1:2. The mass spectral data and elemental analysis (CHNS) also confirmed this assumption.

FTIR analysis: The FTIR spectra (Table-2) were helpful in determining the mode of bond formation between the ligands and the metals. The ligand hydrazone seems to be predominantly present in the enolic rather than the ketonic tautomeric form. This is substantiated by the presence of a broad $\mathrm{OH}$ absorption band for all the ligands (5-8) in the region of 3500-3000 $\mathrm{cm}^{-1}$ which is absent in all the complexes (9-32) pointing to an enolic $\mathrm{O}$ bonding to the metal while there is a discreet shift in the carbonyl frequencies of the hydrazone ligands (5-8) as compared with their corresponding metal complexes. For (7, 2126), the bands in the region of $1550-1490 \mathrm{~cm}^{-1}$ and $1355-1315$ $\mathrm{cm}^{-1}$ correspond to the asymmetric and symmetric stretching of $-\mathrm{NO}_{2}$ group respectively ${ }^{14}$. The absorption bands around 


\begin{tabular}{|c|c|c|c|c|c|c|c|c|}
\hline \multirow{3}{*}{ Compd. } & \multicolumn{7}{|c|}{$\begin{array}{c}\text { TABLE-1 } \\
\text { PHYSICAL DATA FOR THE LIGANDS AND THE METAL COMPLEXES }\end{array}$} & \multirow{3}{*}{$\begin{array}{c}\text { Metal (\%) } \\
\text { Calcd. (found) }\end{array}$} \\
\hline & \multirow{2}{*}{$\begin{array}{l}\text { Yield } \\
(\%)\end{array}$} & \multirow{2}{*}{$\begin{array}{l}\text { m.p. } \\
\left({ }^{\circ} \mathrm{C}\right)\end{array}$} & \multirow{2}{*}{$\mathrm{MS} \dagger$} & \multirow{2}{*}{ m.f. } & \multicolumn{3}{|c|}{ Elemental analysis (\%): Calcd. (Found) } & \\
\hline & & & & & $\mathrm{C}$ & H & $\mathrm{N}$ & \\
\hline 5 & 77.00 & 202 & $366^{* *}$ & $\mathrm{C}_{23} \mathrm{H}_{18} \mathrm{~N}_{4} \mathrm{O}$ & $75.39(75.31)$ & $4.95(4.88)$ & $15.29(15.32)$ & - \\
\hline 9 & 80.50 & $242 *$ & 795 & $\mathrm{C}_{46} \mathrm{H}_{36} \mathrm{~N}_{8} \mathrm{O}_{2} \mathrm{Cu}$ & $69.38(69.33)$ & $4.56(4.52)$ & $14.07(14.08)$ & $7.98(7.90)$ \\
\hline 10 & 83.01 & $237 *$ & 790 & $\mathrm{C}_{46} \mathrm{H}_{36} \mathrm{~N}_{8} \mathrm{O}_{2} \mathrm{Ni}$ & $69.80(69.83)$ & $4.58(4.55)$ & $14.16(14.15)$ & $7.42(7.47)$ \\
\hline 11 & 78.38 & $248^{*}$ & 791 & $\mathrm{C}_{46} \mathrm{H}_{36} \mathrm{~N}_{8} \mathrm{O}_{2} \mathrm{Co}$ & $69.78(69.74)$ & $4.58(4.55)$ & $14.15(14.18)$ & $7.44(7.42)$ \\
\hline 12 & 81.21 & $251^{*}$ & 940 & $\mathrm{C}_{46} \mathrm{H}_{36} \mathrm{~N}_{8} \mathrm{O}_{2} \mathrm{~Pb}$ & $58.77(58.72)$ & $3.86(3.90)$ & $11.92(11.95)$ & $22.04(22.07)$ \\
\hline 13 & 77.88 & $236^{*}$ & 796 & $\mathrm{C}_{46} \mathrm{H}_{36} \mathrm{~N}_{8} \mathrm{O}_{2} \mathrm{Zn}$ & $69.22(69.19)$ & $4.55(4.10)$ & $14.04(14.00)$ & $8.19(8.12)$ \\
\hline 14 & 79.94 & $248^{*}$ & 788 & $\mathrm{C}_{46} \mathrm{H}_{36} \mathrm{~N}_{8} \mathrm{O}_{2} \mathrm{Fe}$ & $70.05(69.10)$ & $4.60(4.07)$ & $14.21(14.14)$ & $7.08(6.53)$ \\
\hline 6 & 85.00 & 208 & $400 * *, 402$ & $\mathrm{C}_{23} \mathrm{H}_{17} \mathrm{~N}_{4} \mathrm{OCl}$ & 68.91 (68.97) & $4.27(4.25)$ & $13.98(13.92)$ & - \\
\hline 15 & 76.00 & $244^{*}$ & 863,865 & $\mathrm{C}_{46} \mathrm{H}_{34} \mathrm{~N}_{8} \mathrm{O}_{2} \mathrm{CuCl}_{2}$ & $63.85(63.89)$ & 3.96 (3.99) & $12.95(12.98)$ & 7.34 (7.39) \\
\hline 16 & 82.09 & $253^{*}$ & 858,860 & $\mathrm{C}_{46} \mathrm{H}_{34} \mathrm{~N}_{8} \mathrm{O}_{2} \mathrm{NiCl}_{2}$ & $64.21(64.27)$ & $3.98(3.94)$ & $13.02(12.95)$ & $6.82(6.89)$ \\
\hline 17 & 81.6 & $271^{*}$ & 859,861 & $\mathrm{C}_{46} \mathrm{H}_{34} \mathrm{~N}_{8} \mathrm{O}_{2} \mathrm{CoCl}_{2}$ & $64.19(64.13)$ & $3.98(3.93)$ & $13.02(13.08)$ & $6.85(6.89)$ \\
\hline 18 & 76.15 & $265^{*}$ & 1008,1010 & $\mathrm{C}_{46} \mathrm{H}_{34} \mathrm{~N}_{8} \mathrm{O}_{2} \mathrm{PbCl}_{2}$ & $54.76(54.71)$ & $3.40(3.47)$ & $11.11(11.19)$ & $20.54(20.48)$ \\
\hline 19 & 81.18 & $243^{*}$ & 864,866 & $\mathrm{C}_{46} \mathrm{H}_{34} \mathrm{~N}_{8} \mathrm{O}_{2} \mathrm{ZnCl}_{2}$ & $63.72(63.79)$ & 3.95 (3.98) & $12.92(12.98)$ & $7.54(7.60)$ \\
\hline 20 & 77.92 & $253^{*}$ & 856,858 & $\mathrm{C}_{46} \mathrm{H}_{34} \mathrm{~N}_{8} \mathrm{O}_{2} \mathrm{FeCl}_{2}$ & $64.43(64.49)$ & $4.00(4.35)$ & $13.07(13.03)$ & $6.51(6.50)$ \\
\hline 7 & 82.00 & 204 & $411 * *$ & $\mathrm{C}_{23} \mathrm{H}_{17} \mathrm{~N}_{5} \mathrm{O}_{3}$ & $67.15(67.09)$ & $4.16(4.11)$ & $17.02(17.08)$ & - \\
\hline 21 & 81.65 & $247 *$ & 885 & $\mathrm{C}_{46} \mathrm{H}_{34} \mathrm{~N}_{10} \mathrm{O}_{6} \mathrm{Cu}$ & $62.33(62.34)$ & $3.87(3.92)$ & $15.80(15.87)$ & 7.17 (7.12) \\
\hline 22 & 79.54 & $242 *$ & 880 & $\mathrm{C}_{46} \mathrm{H}_{34} \mathrm{~N}_{10} \mathrm{O}_{6} \mathrm{Ni}$ & $62.68(62.61)$ & $3.89(3.83)$ & $15.89(15.93)$ & $6.66(6.61)$ \\
\hline 23 & 78.28 & $263 *$ & 881 & $\mathrm{C}_{46} \mathrm{H}_{34} \mathrm{~N}_{10} \mathrm{O}_{6} \mathrm{Co}$ & $62.66(62.61)$ & $3.89(3.87)$ & $15.88(15.82)$ & $6.68(6.64)$ \\
\hline 24 & 73.43 & $257^{*}$ & 1030 & $\mathrm{C}_{46} \mathrm{H}_{34} \mathrm{~N}_{10} \mathrm{O}_{6} \mathrm{~Pb}$ & $53.64(53.61)$ & $3.33(3.31)$ & $13.60(13.67)$ & $20.12(20.08)$ \\
\hline 25 & 76.63 & $248 *$ & 886 & $\mathrm{C}_{46} \mathrm{H}_{34} \mathrm{~N}_{10} \mathrm{O}_{6} \mathrm{Zn}$ & $62.20(62.27)$ & $3.86(3.82)$ & $15.77(15.72)$ & $7.36(7.32)$ \\
\hline 26 & 79.74 & $270 *$ & 878 & $\mathrm{C}_{46} \mathrm{H}_{34} \mathrm{~N}_{10} \mathrm{O}_{6} \mathrm{Fe}$ & $62.88(62.83)$ & $3.90(3.92)$ & 15.94 (15.99) & $6.36(6.32)$ \\
\hline 8 & 82.00 & 201 & $380 * *$ & $\mathrm{C}_{24} \mathrm{H}_{20} \mathrm{~N}_{4} \mathrm{O}$ & 75.77 (75.71) & $5.30(5.25)$ & $14.73(14.69)$ & - \\
\hline 27 & 79.72 & $248^{*}$ & 823 & $\mathrm{C}_{48} \mathrm{H}_{40} \mathrm{~N}_{8} \mathrm{O}_{2} \mathrm{Cu}$ & $69.93(69.87)$ & 4.89 (4.94) & 13.59 (13.54) & 7.71 (7.77) \\
\hline 28 & 78.80 & $255^{*}$ & 818 & $\mathrm{C}_{48} \mathrm{H}_{40} \mathrm{~N}_{8} \mathrm{O}_{2} \mathrm{Ni}$ & 70.34 (70.30) & 4.92 (4.94) & $13.67(13.72)$ & 7.16 (7.10) \\
\hline 29 & 77.44 & $247 *$ & 819 & $\mathrm{C}_{48} \mathrm{H}_{40} \mathrm{~N}_{8} \mathrm{O}_{2} \mathrm{Co}$ & $70.32(70.25)$ & $4.92(4.96)$ & $13.67(13.72)$ & 7.19 (7.23) \\
\hline 30 & 79.31 & $261^{*}$ & 968 & $\mathrm{C}_{48} \mathrm{H}_{40} \mathrm{~N}_{8} \mathrm{O}_{2} \mathrm{~Pb}$ & $59.55(59.60)$ & $4.16(4.10)$ & $11.57(11.51)$ & $21.40(21.47)$ \\
\hline 31 & 79.72 & $252^{*}$ & 824 & $\mathrm{C}_{48} \mathrm{H}_{40} \mathrm{~N}_{8} \mathrm{O}_{2} \mathrm{Zn}$ & 69.77 (69.72) & $4.88(4.83)$ & $13.56(13.60)$ & 7.91 (7.97) \\
\hline 32 & 81.74 & $257 *$ & 816 & $\mathrm{C}_{48} \mathrm{H}_{40} \mathrm{~N}_{8} \mathrm{O}_{2} \mathrm{Fe}$ & 70.59 (70.63) & 4.94 (4.99) & $13.72(13.78)$ & $6.84(6.90)$ \\
\hline
\end{tabular}

TABLE-2

FTIR AND ${ }^{1} \mathrm{H}$ NMR DATA

\begin{tabular}{|c|c|c|}
\hline Compd. & $\operatorname{IR}\left(\mathrm{cm}^{-1}\right)$ & ${ }^{1} \mathrm{H}$ NMR data $\delta(\mathrm{ppm})$ \\
\hline 5 & $3190(\mathrm{OH}), 1641(\mathrm{C}=\mathrm{O}), 1597(\mathrm{C}=\mathrm{N}-\mathrm{N}=\mathrm{C})$ & $\begin{array}{l}10.30(\mathrm{~s}, 1 \mathrm{H}, \mathrm{NHCO}), 8.39(\mathrm{~s}, 1 \mathrm{H}, \mathrm{CH}=\mathrm{N}), 8.34(\mathrm{~s}, 1 \mathrm{H}, \mathrm{Pz}), 7.21-7.85(\mathrm{~m}, 15 \mathrm{H} \text {, } \\
\mathrm{Ar})\end{array}$ \\
\hline 9 & $1672(\mathrm{C}=\mathrm{O}), 1596(\mathrm{C}=\mathrm{N}-\mathrm{N}=\mathrm{C})$ & $8.61(\mathrm{~s}, 1 \mathrm{H}, \mathrm{CH}=\mathrm{N}), 8.36(\mathrm{~s}, 1 \mathrm{H}, \mathrm{Pz}), 7.26-7.91(\mathrm{~m}, 15 \mathrm{H}, \mathrm{Ar})$ \\
\hline 10 & $1642(\mathrm{C}=\mathrm{O}), 1599(\mathrm{C}=\mathrm{N}-\mathrm{N}=\mathrm{C})$ & $8.41(\mathrm{~s}, 1 \mathrm{H}, \mathrm{CH}=\mathrm{N}), 8.14(\mathrm{~s}, 1 \mathrm{H}, \mathrm{Pz}), 7.00-7.71(\mathrm{~m}, 15 \mathrm{H}, \mathrm{Ar})$ \\
\hline 11 & $1650(\mathrm{C}=\mathrm{O}), 1600(\mathrm{C}=\mathrm{N}-\mathrm{N}=\mathrm{C})$ & $8.61(\mathrm{~s}, 1 \mathrm{H}, \mathrm{CH}=\mathrm{N}), 8.31(\mathrm{~s}, 1 \mathrm{H}, \mathrm{Pz}), 7.26-7.89(\mathrm{~m}, 15 \mathrm{H}, \mathrm{Ar})$ \\
\hline 12 & $1641(\mathrm{C}=\mathrm{O}), 1598(\mathrm{C}=\mathrm{N}-\mathrm{N}=\mathrm{C})$ & $8.36(\mathrm{~s}, 1 \mathrm{H}, \mathrm{CH}=\mathrm{N}), 8.22(\mathrm{~s}, 1 \mathrm{H}, \mathrm{Pz}), 7.11-7.77(\mathrm{~m}, 15 \mathrm{H}, \mathrm{Ar})$ \\
\hline 13 & $1642(\mathrm{C}=\mathrm{O}), 1599(\mathrm{C}=\mathrm{N}-\mathrm{N}=\mathrm{C})$ & $8.59(\mathrm{~s}, 1 \mathrm{H}, \mathrm{CH}=\mathrm{N}), 8.33(\mathrm{~s}, 1 \mathrm{H}, \mathrm{Pz}), 7.26-7.90(\mathrm{~m}, 15 \mathrm{H}, \mathrm{Ar})$ \\
\hline 14 & $1672(\mathrm{C}=\mathrm{O}), 1596(\mathrm{C}=\mathrm{N}-\mathrm{N}=\mathrm{C})$ & $8.46(\mathrm{~s}, 1 \mathrm{H}, \mathrm{CH}=\mathrm{N}), 8.28(\mathrm{~s}, 1 \mathrm{H}, \mathrm{Pz}), 7.18-7.84(\mathrm{~m}, 15 \mathrm{H}, \mathrm{Ar})$ \\
\hline 6 & $3160(\mathrm{OH}), 1652(\mathrm{C}=\mathrm{O}), 1599(\mathrm{C}=\mathrm{N}-\mathrm{N}=\mathrm{C})$ & $\begin{array}{l}9.82(\mathrm{~s}, 1 \mathrm{H}, \mathrm{NHCO}), 8.54(\mathrm{~s}, 1 \mathrm{H}, \mathrm{CH}=\mathrm{N}), 8.35(\mathrm{~s}, 1 \mathrm{H}, \mathrm{Pz}), 7.25-7.91(\mathrm{~m}, 14 \mathrm{H} \\
\mathrm{Ar})\end{array}$ \\
\hline 15 & $1670(\mathrm{C}=\mathrm{O}), 1597(\mathrm{C}=\mathrm{N}-\mathrm{N}=\mathrm{C})$ & $8.68(\mathrm{~s}, 1 \mathrm{H}, \mathrm{CH}=\mathrm{N}), 8.27(\mathrm{~s}, 1 \mathrm{H}, \mathrm{NCH}=), 7.34-7.83(\mathrm{~m}, 14 \mathrm{H}, \mathrm{Ar})$ \\
\hline 16 & $1656(\mathrm{C}=\mathrm{O}), 1599(\mathrm{C}=\mathrm{N}-\mathrm{N}=\mathrm{C})$ & $8.69(\mathrm{~s}, 1 \mathrm{H}, \mathrm{CH}=\mathrm{N}), 8.28(\mathrm{~s}, 1 \mathrm{H}, \mathrm{Pz}), 7.26-7.86(\mathrm{~m}, 14 \mathrm{H}, \mathrm{Ar})$ \\
\hline 17 & $1656(\mathrm{C}=\mathrm{O}), 1605(\mathrm{C}=\mathrm{N}-\mathrm{N}=\mathrm{C})$ & $8.66(\mathrm{~s}, 1 \mathrm{H}, \mathrm{CH}=\mathrm{N}), 8.23(\mathrm{~s}, 1 \mathrm{H}, \mathrm{Pz}), 7.26-7.88(\mathrm{~m}, 14 \mathrm{H}, \mathrm{Ar})$ \\
\hline 18 & $1656(\mathrm{C}=\mathrm{O}), 1601(\mathrm{C}=\mathrm{N}-\mathrm{N}=\mathrm{C})$ & $8.59(\mathrm{~s}, 1 \mathrm{H}, \mathrm{CH}=\mathrm{N}), 8.31(\mathrm{~s}, 1 \mathrm{H}, \mathrm{Pz}), 7.26-7.89(\mathrm{~m}, 14 \mathrm{H}, \mathrm{Ar})$ \\
\hline 19 & $1649(\mathrm{C}=\mathrm{O}), 1592(\mathrm{C}=\mathrm{N}-\mathrm{N}=\mathrm{C})$ & $8.61(\mathrm{~s}, 1 \mathrm{H}, \mathrm{CH}=\mathrm{N}), 8.29(\mathrm{~s}, 1 \mathrm{H}, \mathrm{Pz}), 7.25-7.90(\mathrm{~m}, 14 \mathrm{H}, \mathrm{Ar})$ \\
\hline 20 & $1649(\mathrm{C}=\mathrm{O}), 1598(\mathrm{C}=\mathrm{N}-\mathrm{N}=\mathrm{C})$ & $8.63(\mathrm{~s}, 1 \mathrm{H}, \mathrm{CH}=\mathrm{N}), 8.39(\mathrm{~s}, 1 \mathrm{H}, \mathrm{Pz}), 7.24-7.91(\mathrm{~m}, 14 \mathrm{H}, \mathrm{Ar})$ \\
\hline 7 & $\begin{array}{l}3210(\mathrm{OH}), 1649(\mathrm{C}=\mathrm{O}), 1597(\mathrm{C}=\mathrm{N}-\mathrm{N}=\mathrm{C}), 1502 \& \\
1334\left(\mathrm{NO}_{2}\right)\end{array}$ & $\begin{array}{l}10.34(\mathrm{~s}, 1 \mathrm{H}, \mathrm{NHCO}), 8.49(\mathrm{~s}, 1 \mathrm{H}, \mathrm{CH}=\mathrm{N}), 8.41(\mathrm{~s}, 1 \mathrm{H}, \mathrm{Pz}), 7.11-7.93(\mathrm{~m}, 14 \mathrm{H} \text {, } \\
\mathrm{Ar})\end{array}$ \\
\hline 21 & $1641(\mathrm{C}=\mathrm{O}), 1598(\mathrm{C}=\mathrm{N}-\mathrm{N}=\mathrm{C}), 1492 \& 1343\left(\mathrm{NO}_{2}\right)$ & $8.40(\mathrm{~s}, 1 \mathrm{H}, \mathrm{CH}=\mathrm{N}), 8.29(\mathrm{~s}, 1 \mathrm{H}, \mathrm{Pz}), 7.25-7.88(\mathrm{~m}, 14 \mathrm{H}, \mathrm{Ar})$ \\
\hline 22 & $1640(\mathrm{C}=\mathrm{O}), 1598(\mathrm{C}=\mathrm{N}-\mathrm{N}=\mathrm{C}), 1501 \& 1341\left(\mathrm{NO}_{2}\right)$ & $8.46(\mathrm{~s}, 1 \mathrm{H}, \mathrm{CH}=\mathrm{N}), 8.29(\mathrm{~s}, 1 \mathrm{H}, \mathrm{Pz}), 7.26-7.88(\mathrm{~m}, 14 \mathrm{H}, \mathrm{Ar})$ \\
\hline 23 & $1646(\mathrm{C}=\mathrm{O}), 1597(\mathrm{C}=\mathrm{N}-\mathrm{N}=\mathrm{C}), 1500 \& 1335\left(\mathrm{NO}_{2}\right)$ & $8.46(\mathrm{~s}, 1 \mathrm{H}, \mathrm{CH}=\mathrm{N}), 8.27(\mathrm{~s}, 1 \mathrm{H}, \mathrm{Pz}), 7.26-7.87(\mathrm{~m}, 14 \mathrm{H}, \mathrm{Ar})$ \\
\hline 24 & $1651(\mathrm{C}=\mathrm{O}), 1597(\mathrm{C}=\mathrm{N}-\mathrm{N}=\mathrm{C}), 1501 \& 1334\left(\mathrm{NO}_{2}\right)$ & $8.45(\mathrm{~s}, 1 \mathrm{H}, \mathrm{CH}=\mathrm{N}), 8.29(\mathrm{~s}, 1 \mathrm{H}, \mathrm{Pz}), 7.26-7.88(\mathrm{~m}, 14 \mathrm{H}, \mathrm{Ar})$ \\
\hline 25 & $1651(\mathrm{C}=\mathrm{O}), 1598(\mathrm{C}=\mathrm{N}-\mathrm{N}=\mathrm{C}), 1500 \& 1339\left(\mathrm{NO}_{2}\right)$ & $8.43(\mathrm{~s}, 1 \mathrm{H}, \mathrm{CH}=\mathrm{N}), 8.30(\mathrm{~s}, 1 \mathrm{H}, \mathrm{Pz}), 7.26-7.91(\mathrm{~m}, 14 \mathrm{H}, \mathrm{Ar})$ \\
\hline 26 & $1681(\mathrm{C}=\mathrm{O}), 1596(\mathrm{C}=\mathrm{N}-\mathrm{N}=\mathrm{C}), 1502 \& 1341\left(\mathrm{NO}_{2}\right)$ & $8.57(\mathrm{~s}, 1 \mathrm{H}, \mathrm{CH}=\mathrm{N}), 8.30(\mathrm{~s}, 1 \mathrm{H}, \mathrm{Pz}), 7.18-7.89(\mathrm{~m}, 14 \mathrm{H}, \mathrm{Ar})$ \\
\hline 8 & $3237(\mathrm{OH}), 1640(\mathrm{C}=\mathrm{O}), 1604(\mathrm{C}=\mathrm{N}-\mathrm{N}=\mathrm{C})$ & $\begin{array}{l}9.71(\mathrm{~s}, 1 \mathrm{H}, \mathrm{NHCO}), 8.57(\mathrm{~s}, 1 \mathrm{H}, \mathrm{CH}=\mathrm{N}), 8.30(\mathrm{~s}, 1 \mathrm{H}, \mathrm{Pz}), 7.18-7.89(\mathrm{~m}, 14 \mathrm{H} \\
\mathrm{Ar}), 2.35\left(\mathrm{~s}, 3 \mathrm{H}, \mathrm{CH}_{3}\right)\end{array}$ \\
\hline 27 & $1671(\mathrm{C}=\mathrm{O}), 1597(\mathrm{C}=\mathrm{N}-\mathrm{N}=\mathrm{C})$ & $8.61(\mathrm{~s}, 1 \mathrm{H}, \mathrm{CH}=\mathrm{N}), 8.41(\mathrm{~s}, 1 \mathrm{H}, \mathrm{Pz}), 7.12-7.93(\mathrm{~m}, 14 \mathrm{H}, \mathrm{Ar}) 2.35\left(\mathrm{~s}, 3 \mathrm{H}, \mathrm{CH}_{3}\right)$ \\
\hline 28 & $1641(\mathrm{C}=\mathrm{O}), 1600(\mathrm{C}=\mathrm{N}-\mathrm{N}=\mathrm{C})$ & $8.60(\mathrm{~s}, 1 \mathrm{H}, \mathrm{CH}=\mathrm{N}), 8.29(\mathrm{~s}, 1 \mathrm{H}, \mathrm{Pz}), 7.22-7.85(\mathrm{~m}, 14 \mathrm{H}, \mathrm{Ar}) 2.35\left(\mathrm{~s}, 3 \mathrm{H}, \mathrm{CH}_{3}\right)$ \\
\hline 29 & $1642(\mathrm{C}=\mathrm{O}), 1607(\mathrm{C}=\mathrm{N}-\mathrm{N}=\mathrm{C})$ & $8.63(\mathrm{~s}, 1 \mathrm{H}, \mathrm{CH}=\mathrm{N}), 8.45(\mathrm{~s}, 1 \mathrm{H}, \mathrm{Pz}), 7.20-7.76(\mathrm{~m}, 14 \mathrm{H}, \mathrm{Ar}) 2.37\left(\mathrm{~s}, 3 \mathrm{H}, \mathrm{CH}_{3}\right)$ \\
\hline 30 & $1641(\mathrm{C}=\mathrm{O}), 1600(\mathrm{C}=\mathrm{N}-\mathrm{N}=\mathrm{C})$ & $8.55(\mathrm{~s}, 1 \mathrm{H}, \mathrm{CH}=\mathrm{N}), 8.34(\mathrm{~s}, 1 \mathrm{H}, \mathrm{Pz}), 7.16-7.90(\mathrm{~m}, 14 \mathrm{H}, \mathrm{Ar}) 2.33\left(\mathrm{~s}, 3 \mathrm{H}, \mathrm{CH}_{3}\right)$ \\
\hline 31 & $1641(\mathrm{C}=\mathrm{O}), 1600(\mathrm{C}=\mathrm{N}-\mathrm{N}=\mathrm{C})$ & $8.56(\mathrm{~s}, 1 \mathrm{H}, \mathrm{CH}=\mathrm{N}), 8.30(\mathrm{~s}, 1 \mathrm{H}, \mathrm{Pz}), 7.17-7.73(\mathrm{~m}, 14 \mathrm{H}, \mathrm{Ar}) 2.33\left(\mathrm{~s}, 3 \mathrm{H}, \mathrm{CH}_{3}\right)$ \\
\hline 32 & $1642(\mathrm{C}=\mathrm{O}), 1598(\mathrm{C}=\mathrm{N}-\mathrm{N}=\mathrm{C})$ & $8.51(\mathrm{~s}, 1 \mathrm{H}, \mathrm{CH}=\mathrm{N}), 8.42(\mathrm{~s}, 1 \mathrm{H}, \mathrm{Pz}), 7.15-7.92(\mathrm{~m}, 14 \mathrm{H}, \mathrm{Ar}), 2.33\left(\mathrm{~s}, 3 \mathrm{H}, \mathrm{CH}_{3}\right)$ \\
\hline
\end{tabular}


$1660-1590 \mathrm{~cm}^{-1}$ are designated to the azomethine $(\mathrm{C}=\mathrm{N}-\mathrm{N}=\mathrm{C})$ group $^{15}$. In the complexes, the frequencies of azomethine groups appear mostly towards the lower region which strongly indicates that the ligands coordinate to the metal through the carbonyl oxygen and the azomethine nitrogen of the enolic form of the ligand (Fig. 1). This is further confirmed on the basis of ${ }^{1} \mathrm{H}$ NMR studies (see below). Hence, the ligands act as monobasic bidentate ligands in all the complexes.
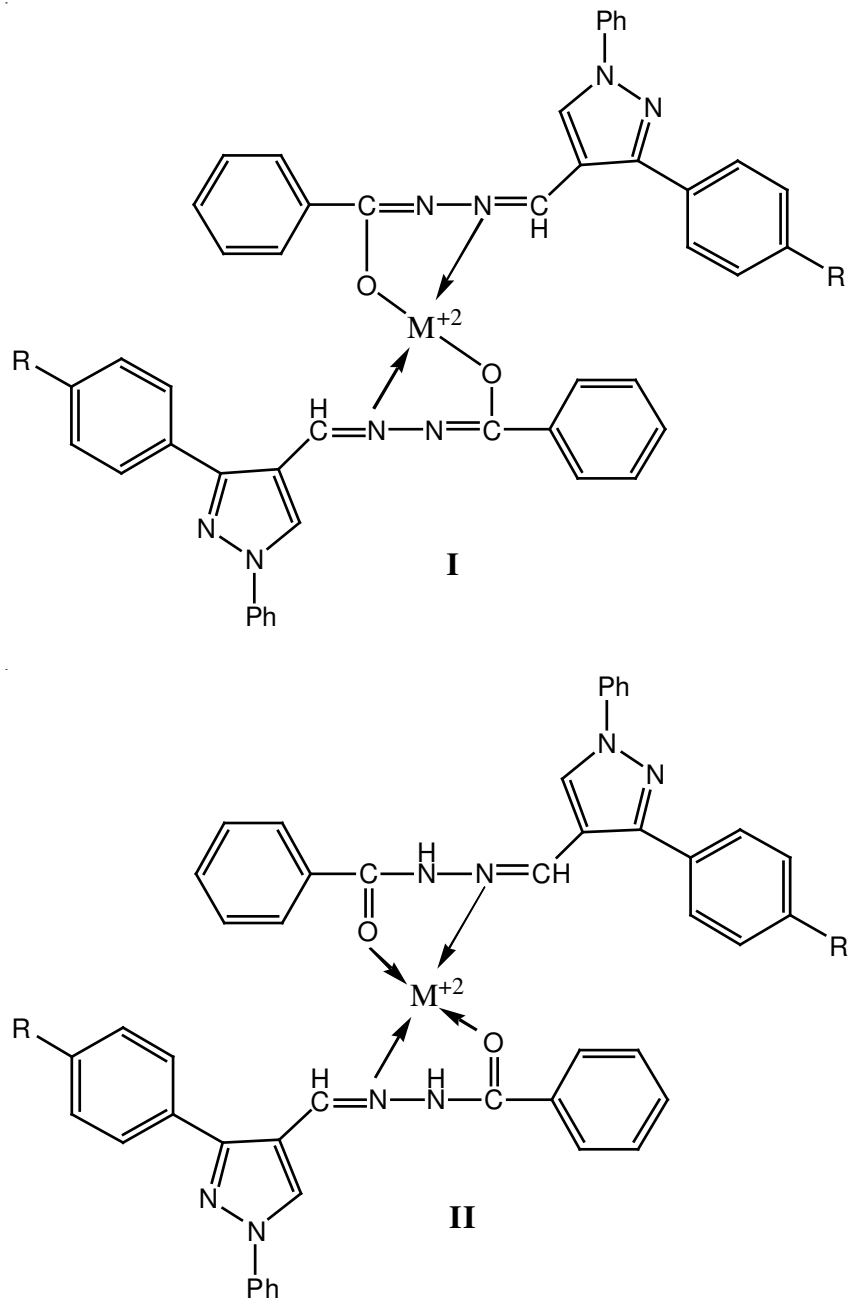

Fig. 1. Structure of metal complexes (9-32), $\mathrm{R}=\mathrm{H}, \mathrm{Cl}, \mathrm{NO}_{2}, \mathrm{CH}_{3}$

${ }^{1}$ H NMR spectra: The chemical shift values for the ligands (5-8) and the metal complexes are recorded in Table2 . All the ligands differ only in substitution at $4^{\prime}$-position on the 3-aryl substituent in the pyrazole $(\mathrm{Pz})$ ring. The 3-aryl ring protons show multiplets in the 7.11-7.93 ppm region. The characteristic NHCO signal, observed at $10.30 \mathrm{ppm}$ (5), is conspicuously absent in the all the metal complexes (9-32). It was further observed that the signals of the protons at $\mathrm{NHCO}$, $\mathrm{H}-5$ proton of pyrazole $(\mathrm{Pz})$ and 4 '-substituted phenyl ring were shifted from their original $\delta$ values from the respective ligands (Table-2). Thus in the formation of complexes of these bivalent metal ions, the NHCO signals disappeared due to enolization of the ligands during complex formation with the help of the carbonyl oxygen and azomethine nitrogen of the enolic tautomer I (Fig. 1).
Electron spray ionization mass spectra: The positive ion ESI MS of the ligands (5-8) and their metal complexes (9-32) in $\mathrm{MeOH}$ are dominated by the parent molecular ion peaks. The $\mathrm{m} / \mathrm{z}$ values shown in Table- 1 are $\mathrm{L}^{+}$molecular ion peak for ligands ( $\mathrm{L}$ for ligands) and monopositive complex ion $\left[\mathrm{ML}_{2}+2 \mathrm{H}\right]^{+}$molecular ion peaks. The data support the assumption that metal to ligand ratio is 1:2 in all the complexes.

\section{Biological activities}

Antibacterial activity: The method adopted here is based on the principle that microbial cell number increases as the microbial growth proceeds in the log phase of growth which results in the increased absorbance of the broth medium ${ }^{16,17}$. Bacillus subtills was the bacterial strain used for the present study. Ampicillin was used as the positive control and solvent/ nutrient broth as the negative control. The per cent inhibitions of the synthesized compounds, in comparison with ampicillin, were recorded in Table-3.

\begin{tabular}{|c|c|c|}
\hline \multicolumn{3}{|c|}{$\begin{array}{c}\text { TABLE-3 } \\
\text { BIOLOGICAL ACTIVITIES }\end{array}$} \\
\hline Compd. & $\begin{array}{c}\text { Antibacterial activity* } \\
\text { Bacillus subtills } \\
\text { (Inhibition \%) }\end{array}$ & $\begin{array}{l}\text { Antioxidant activity** } \\
\text { (Inhibition \%) }\end{array}$ \\
\hline 5 & 10.75 & 31.48 \\
\hline 9 & 8.62 & 10.38 \\
\hline 10 & 13.46 & 39.74 \\
\hline 11 & 27.58 & 55.58 \\
\hline 12 & 15.35 & 18.44 \\
\hline 13 & 12.47 & 23.37 \\
\hline 14 & 12.56 & 03.11 \\
\hline 6 & 32.09 & 31.21 \\
\hline 15 & 16.91 & 74.00 \\
\hline 16 & 12.72 & 12.98 \\
\hline 17 & 16.25 & 65.45 \\
\hline 18 & 14.69 & 52.20 \\
\hline 19 & 32.51 & 17.40 \\
\hline 20 & 6.15 & 71.94 \\
\hline 7 & 17.78 & 33.33 \\
\hline 21 & 44.83 & 18.44 \\
\hline 22 & 42.69 & 28.31 \\
\hline 23 & 36.20 & 15.00 \\
\hline 24 & 11.82 & 76.88 \\
\hline 25 & 13.79 & 33.24 \\
\hline 26 & 3.61 & 68.31 \\
\hline 8 & 20.67 & 14.81 \\
\hline 27 & 28.98 & 7.27 \\
\hline 28 & 36.29 & 11.94 \\
\hline 29 & 33.66 & 11.16 \\
\hline 30 & 5.17 & 84.67 \\
\hline 31 & 3.44 & 84.69 \\
\hline 32 & 2.87 & 83.89 \\
\hline
\end{tabular}

It is clear that most of the compounds showed significant antibacterial activities. In case of ligand (5) and its metal complexes, the metal complexation caused the activity to increase except for $\mathrm{Cu}$ complex (9), where activity decreased to some extent (10.75 to $8.62 \%$ ). The maximum activity was found in cobalt complex (11) (27.58\%). For ligand (6) ( $4^{\prime}$-chloro derivative) and its metal complexes, the complex 
formation caused the activity to decrease. The zinc complex (19) had almost similar activity as that of the ligand. In (7) (4'-nitro derivative) and its complexes with metals, the antibacterial activity was increased in complexes with $\mathrm{Cu}(\mathbf{2 1})$, $\mathrm{Ni}$ (22) and $\mathrm{Co}(\mathbf{2 3})$, whereas it decreased in complexes with $\mathrm{Pb}$ (24), Zn (25) and Fe (26). Similarly for (8) (4'-methyl derivative) and the complexes, it was found that the activity increased with $\mathrm{Cu}$ (27), $\mathrm{Ni}(\mathbf{2 8})$ and $\mathrm{Co}$ (29) complexes whereas it decreased in complexes with $\mathrm{Pb}(\mathbf{3 0}), \mathrm{Zn}(\mathbf{3 1})$ and $\mathrm{Fe}(\mathbf{3 2})$ to a significant extent.

When the activities of the different ligands and their complexes are compared, very interesting results are obtained. In ligands, on substitution of $\mathrm{H}$ with $-\mathrm{Cl},-\mathrm{NO}_{2}$ or $-\mathrm{CH}_{3}$ at $4^{\prime}$ position in the parent ligand (5), the antibacterial activity increased to significant extent. So, the presence of some group at the specified position causes an increase in the activity; this increase is greater in case of small sized group $(-\mathrm{Cl})$ as compared to large ones.

In $\mathrm{Cu}$ complexes $(\mathbf{9}, \mathbf{1 5}, \mathbf{2 1}, \mathbf{2 7})$ of all four ligands, the complex with a $-\mathrm{NO}_{2}$ group at 4 '-position (7) showed the maximum activity $(44.83 \%)$ of all the complexes. In $\mathrm{Ni}$ complexes, (22) showed the second maximum activity (42.83 $\%$ ). The similar result was observed in Co complex (23) (ligand with nitro group). The complexes of $\mathrm{Pb}$ (12) and $\mathrm{Fe}(\mathbf{1 4})$ showed the best results (highest activities) with ligand having no group at 4 '-position, rather the activities were decreased with any of the substitution with electron withdrawing or electron donating group. The $\mathrm{Zn}$ complexes showed a very different behaviour. The $-\mathrm{Cl}$ substituted ligand showed the maximum activity.

It can be concluded that the complexes of metal with ligand having the electron withdrawing group $\left(-\mathrm{NO}_{2}\right)$ were the most active against Bacillus subtills.

Antioxidant activity: Garret nitric oxide (NO) scavenging method was used for the determination of antioxidant activity ${ }^{18}$. The method is based on the principle that nitrate present in the sample must be reduced to nitrite. This method involves the determination of nitrite, instead of nitrate in the presence of Griss reagent. The results are presented in Table-3.

The antioxidant activity of ligand (5) was substantially increased on complexation with $\mathrm{Ni}(\mathbf{1 0})$ and $\mathrm{Co}(\mathbf{1 1})$ from 31.48 to 39.74 and 55.58, respectively. The complex with $\mathrm{Fe}$ (14) seemed to be the least active, almost 10 times less than the ligand. With $4^{\prime}$-chloro substituted ligand (6), the activity increased with $\mathrm{Cu}$ (15), $\mathrm{Co}(\mathbf{1 7}), \mathrm{Pb}(\mathbf{1 8})$ and $\mathrm{Fe}(\mathbf{2 0})$ complexes whereas the activity of $\mathrm{Ni}(\mathbf{1 6})$ and $\mathrm{Zn}(\mathbf{1 9})$ complexes was quite less than that of ligand (6). For $4^{\prime}$-nitro ligand (7), $\mathrm{Pb}(\mathbf{2 4})$ and $\mathrm{Fe}(\mathbf{2 6})$ complex formation increased the antioxidant behaviour of the compound and the complexes with other four metals $(\mathrm{Cu}(\mathbf{2 1}), \mathrm{Ni}(\mathbf{2 2}), \mathrm{Co}(\mathbf{2 3})$ and $\mathrm{Zn}(\mathbf{2 5}))$ caused the activity to decrease significantly. Similarly for 4'-methyl substituted ligand $(\mathbf{8})$, the activity increased after complex formation with $\mathrm{Pb}(\mathbf{3 0}), \mathrm{Zn}(\mathbf{3 1})$ and $\mathrm{Fe}(\mathbf{3 2})$ : the $\mathrm{Zn}(\mathbf{3 1})$ complex showed the maximum antioxidant activity of all the compounds studied.

Among all the ligands (5-8), it was found that the substitution at $4^{\prime}$-position with electron withdrawing bulky group $-\mathrm{NO}_{2}$ caused the antioxidant activity to increase whereas the presence of electron donating bulky group $-\mathrm{CH}_{3}$ decreased the activity. In the complexes, the $\mathrm{Cu}(\mathbf{1 5})$ and $\mathrm{Co}(\mathbf{1 7})$ complexes with chloro substituted ligand showed the maximum activity. Among Ni complexes, (10) exhibited the maximum activity. It was found that the $\mathrm{Pb}(\mathbf{3 0}), \mathrm{Zn}(\mathbf{3 1})$ and $\mathrm{Fe}(\mathbf{3 2})$ complexes with the $4^{\prime}$-methyl substituted ligand exhibited the highest values among all the compounds/complexes studied.

\section{Conclusion}

The present study revealed the complexes formed from ligands (5-8) have stoichiometry of 2:1 (ligand: metal) with a possible structure showing chelation through carbonyl oxygen and azomethyne nitrogen. These are evidenced by their IR, ${ }^{1} \mathrm{H}$ NMR and elemental analytical data. The antibacterial activity against Bacillus subtills showed that within a series, the metal complexes are more active than corresponding ligands. In ligands, on substitution of $\mathrm{H}$ with $-\mathrm{Cl},-\mathrm{NO}_{2}$ or $-\mathrm{CH}_{3}$ at $4^{\prime}$-position, the antibacterial activity increased significantly. The complexes of metals with ligands having electron withdrawing group $\left(-\mathrm{NO}_{2}\right)$ were the most active against the gram positive bacteria. The antioxidant behaviour of the ligands having $-\mathrm{NO}_{2}$ group increased than that with $-\mathrm{H}$ whereas the presence of electron donating bulky group $-\mathrm{CH}_{3}$ decreased the activity. The $\mathrm{Cu}$ and $\mathrm{Co}$ complexes with chloro substituted ligand showed the maximum activity. It was found that $\mathrm{Pb}, \mathrm{Zn}$ and Fe complexes with $4^{\prime}$-methyl substituted ligand exhibited the highest values among all studied compounds.

\section{ACKNOWLEDGEMENTS}

One of the authors (MN) acknowledged the financial support by HEC Pakistan in the form of an indigenous $\mathrm{Ph} . \mathrm{D}$. fellowship and assistance in the form of IRSIP to travel and work at Australian National University. The author also acknowledged the helpful discussion with Prof. Dr. Saeed Ahmed (University of Engineering \& Technology, Lahore).

\section{REFERENCES}

1. D.L. Klayman, J.F. Bartosevich, T.S. Griffin, C.J. Mason and J.P. Scovill, J. Med. Chem., 22, 855 (1979).

2. D.X. West, S.B. Padhye and P.B. Sonawane, Struct. Bond., 76, 1 (1991).

3. A.E. Liberta and D.X. West, Biometals, 5, 121 (1992).

4. F.A. French, E.J. Blanz, J.R. Do Amaral and D.A. French, J. Med. Chem., 13, 1117 (1970).

5. H. Beraldo and D. Gambino, Mini Rev. Chem., 4, 31 (2004).

6. S. Rollas and S.G. Kucukguzel, Molecules, 12, 1910 (2007).

7. E.W. Ainscough, A.M. Brodie, W.A. Denny, G.J. Finlay, S.A. Gothe and J.D. Ranford, J. Inorg. Biochem., 77, 125 (1999).

8. D. Van Reyk, S. Sarel and N. Hunt, Biochem. Pharmacol., 60, 581 (2000).

9. I. Ahmad, M.A. Khan and M. Ather, Pak. J. Sci. Ind. Res., 43, 38 (2000).

10. I. Ahmad, M.A. Khan and M. Ather, J. Pure Appl. Sci., 17, 67 (1998).

11. I. Ahmad, M. A. Khan, M. Ather, Pak. J. Sci. Ind. Res., 44, 268 (2001)

12. O. Prakash, K. Pannu and A. Kumar, Molecules, 11, 43 (2006).

13. V.M. Leovac, S.B. Novakovic, G.A. Bogdanovic, M.D. Joksovic, K.M. Szecsenyi and V.M. Cesljevic, Polyhedron, 26, 3783 (2007).

14. D.L. Pavia, G.M. Lampman and G.S. Kriz, Introduction to Spectroscopy, Thomson Learning Inc. USA (2001).

15. R.T. Conley, Infrared Spectroscopy, Allyn and Bacom Inc. Boston, USA (1972).

16. M. Kaspady, V.K. Narayanaswamy, M. Raju and G.K. Rao, Lett. Drug Design Discov., 6, 21 (2009).

17. A.K. Patel, R.J. Patel, K.H. Patel and R.M. Patel, J. Chil. Chem. Soc., 54, 228 (2009).

18. D.C. Garret, Quantitative Analysis of Drugs, Chapman and Hall, Japan, Vol. 3, p. 456 (1964). 